

\title{
Screening of newly-diagnosed adults with Tuberculosis for Diabetes mellitus and Impaired Glucose Tolerance in a Local Government Area in Ogun State, Nigeria
}

\author{
Salako AA*1, Adenowo OA², Amoran OE ${ }^{1}$, Odusan $\mathrm{O}^{3}$, Mautin GJ ${ }^{1}$, \\ Sanya-Ishijola FA ${ }^{4}$ \\ 1Department of Community Medicine \& Primary Care, Obafemi Awolowo College of Health Sciences, Olabisi \\ Onabanjo University, Sagamu, Nigeria \\ 2Olabisi Onabanjo University Health Centre, Ago-Iwoye, Nigeria \\ ${ }^{3}$ Department of Medicine, Obafemi Awolowo College of Health Sciences, Olabisi Onabanjo University, Sagamu, \\ Nigeria \\ ${ }^{4}$ Community Medicine and Primary Care, Olabisi Onabanjo University Teaching Hospital, Sagamu, Nigeria \\ *Correspondence: Dr. AA Salako, Department of Community Medicine and Primary Care, Obafemi Awolowo \\ College of Health Sciences, Olabisi Onabanjo University, Sagamu, Ogun State, Nigeria. \\ Email: adesalako768@gmail.com; ORCID - https://orcid.org/0000-0003-4179-2182
}

\begin{abstract}
Background: Emerging patterns in epidemiological transitions have led to increased risk for Non-Communicable Diseases and infectious conditions globally. Diabetes mellitus (DM) in a population is one such risk factor that could lead to the development of Tuberculosis (TB) due to weakened immune functions in affected persons, with implications for mortality. If not diagnosed early, DM also leads to poor treatment outcomes in TB.

Objective: To assess the prevalence of DM and impaired glucose tolerance (pre-diabetes) among newly diagnosed tuberculosis cases in Ijebu-Ode Local Government Area of Ogun State, Nigeria.

Methods: This cross-sectional study was conducted among 100 newly diagnosed Pulmonary TB patients in ljebu-Ode LGA. The patients were selected from four health facilities and were screened for elevated Fasting Blood Glucose levels using a laboratory spectrophotometer (glucose oxidase method).

Results: The mean age of the 100 study participants was 33 years $( \pm 9.82)$, with ages within $19-62$ years. Eleven $(11.0 \%)$ had elevated blood glucose levels suggestive of DM, while $20(20.0 \%)$ had impaired fasting blood glucose levels. Among these 31 patients, 18 (58.0\%) were males while $13(42.0 \%)$ were females. The modal ages were within 20-39 years.

Conclusion: Screening for DM by healthcare providers should be routinely conducted before commencing TB patients on treatment. This will aid early detection, improve treatment outcomes of TB and prevent mortality among patients with these co-morbidities.
\end{abstract}

Keywords: Diabetes mellitus, Directly-Observed Therapy Short Course (DOTS), Screening, Tuberculosis. 


\section{Introduction}

The world now has a huge burden of both infectious and Non-Communicable Diseases (NCDs), with varying distributions due to many factors, including levels of socioeconomic development of countries and notable demographic and epidemiological transitions. [1] The frequency of these disease groups existing as co-morbidities, especially those with the potential to depress immune functions and lead to death, such as with Diabetes mellitus (DM) and $\mathrm{TB}$, is a growing cause for concern. [2] $\mathrm{DM}$ is a chronic NCD, characterized by hyperglycaemia which results from defects in insulin secretion, insulin action or both. Insulin, as a hormone, regulates blood glucose levels, but this function is impaired in DM leading to elevated blood glucose levels. This blood glucose derangement causes serious damage to many organ systems in the body, especially the nerves and blood vessels.

There are two major subtypes of this disease namely; Type 1 Insulin-Dependent Diabetes Mellitus (IDDM) and Non-Insulin Dependent Diabetes Mellitus (NIDDM). The latter is more common globally. The symptoms in both types include excessive urination in large quantities (polyuria), thirst and excessive water intake (polydipsia), frequent hunger, weight loss, visual changes, and fatigue. Pre-diabetes, on the other hand, is an intermediate condition in the transition between normality and DM and this includes impaired fasting blood glucose or impaired glucose tolerance which comprises elevated blood glucose levels, though not high enough to be classified as DM. The patients with such levels of elevated blood glucose in prediabetes are still at risk of progression to DM.

Currently, 387 million adults aged 20-79 years of the world's 7 billion population have DM, ${ }^{[3]}$ and it is predicted that the global DM prevalence will increase by $50 \%$ by the year 2030. [4] Also, over $80 \%$ of diabetes deaths occur in low- and middle-income countries (LMICs). In Africa, the World Health Organisation (WHO) estimated that the prevalence rates for the disease have increased more in the LMICs than in highincome countries. [5] The WHO Fact Sheets for the year 2014 showed an estimated 25 million people in sub-Saharan Africa (SSA) lived with $\mathrm{DM}$, an increase from the 4 million people in 1980; this has also been projected to increase up to 41.5 million by the year 2035 .

The prevalence of DM in SSA countries is estimated to be about $1 \%$ in rural regions, while it varies (5-7\%) for urban settings. [6] Generally, accurate country data in SSA, including Nigeria, are lacking, and this results in the use of rough estimates because of a lack of Diabetes Registries and poor data collection on the continent. This is corroborated by conflicting reports and statistics on Nigeria; according to the International Diabetes Federation (IDF), the prevalence of DM is about 2\% (IDF Diabetes Atlas 2017). [7] This may be viewed from a perspective that even though these statistics were quoted, yet it is significant when one considers Nigeria's continuously growing large population and that about 4 million people are noted to have had DM especially when compared with other countries with lesser population estimates. This is further supported by facts that Nigeria had the highest proportion of people with DM in Africa in the year 2013. [8] There are also suggestions that a large number of cases and children are unaccounted for. [ 8,9] This may further account for a range of prevalence rates for DM in the literature in Nigeria's general population estimated at $8-10 \%$. [10] A systematic review was done in Nigeria and it also showed a prevalence of less than $10 \%$ for the year 2013. [11] In the same vein, the prevalence of impaired glucose tolerance in Nigeria is also estimated at $8.9 \%$. [7] 
In all forms of DM, cell-mediated immunity is depressed due to the hyperglycaemic state. [12] This could serve as a background for the development of Tuberculosis (TB), which is also a chronic disease and remains a major cause of mortality and morbidity in developing countries. For instance, people with DM have been found to have a three-fold increase in the risk of developing active $\mathrm{TB}$ due to impaired immunity. [13,14] While DM and TB may exist as co-morbidities, according to some studies, TB could be involved as a risk factor for the onset of DM. ${ }^{[15,16]}$ The prevalence of DM among TB patients is often noted to be higher when compared to the general population, with a prevalence that could range from between 1.9\% to $45 \%$, [16, 17] depending on regions, and with a global median prevalence of $16 \% .[18]$ Furthermore, in terms of infectious disease control efforts, TB often worsens glycaemic control in concurrent cases of DM, with worse tuberculosis treatment outcomes, a higher rate of relapse following tuberculosis treatment, and a higher risk of death than patients with tuberculosis alone. [19]

Given these facts, and the presence of global transitions involving Non-Communicable Diseases and infectious diseases, such as the relationship between $\mathrm{TB}$ and $\mathrm{DM}$, now being postulated as global co-epidemics; more evidence is required to understand the relationship and burden of these diseases in different geographical settings. The primary objective of this study was the determination of the prevalence of DM and impaired fasting glucose tolerance states among patients with established TB, and to highlight the significance of DM screening among TB patients on Direct Observed Therapy Short-course (DOTS) treatment in a semi-urban centre in Ijebu-Ode Local Government Area (LGA).

\section{Methods}

Study area

This study was carried out at Ijebu-Ode in Ogun-State, South-west Nigeria, on the LagosSagamu-Benin highway. The city is located 110 $\mathrm{km}$ by road, north-east of Lagos within $100 \mathrm{~km}$ of the Atlantic ocean in the eastern part of Ogun State with a warm tropical climate. There are ten school health services, one general hospital and about nine health posts/centres located within the LGA. There were four DOTS facilities in the town. Cases seen at the health centres are Drug Sensitive TB cases. The population of the town is 157,161 [20] and the residents are mainly people of Ijebu descent; most of whom are traders with some engaging in farming.

\section{Study Population}

Patients diagnosed with Pulmonary Tuberculosis and managed on DirectlyObserved Therapy Short Course (DOTS) at selected primary health care facilities in IjebuOde LGA, constituted the study population. Only those who were resident within Ijebu-Ode LGA were eligible for participation in the study.

\section{Study design}

A cross-sectional descriptive study was carried out among patients diagnosed with pulmonary TB in ljebu-Ode LGA, Ogun State, between January and June 2008, at health centres which offered DOTS services

\section{Sampling technique}

The total sample of all patients presenting at the facilities offering DOTS services was used. There were only four health facilities that offer DOTS services out of the nine health centres in the local government. Consecutive recruitment of clients was carried out at each facility within the period of the study. A sample size of 100 persons was earlier calculated using a 
prevalence of $5 \%$ and this target was achieved during the four months of data collection.

\section{Study Instruments}

A semi-structured, interviewer-administered questionnaire was used for data collection. The questionnaire was made up of four sections covering respondents' socio-demographic characteristics, family and social history, duration of symptoms, presenting complaints, past medical history including a history of DM. The questionnaire was pretested in a health facility at Ikenne LGA.

Spectrophotometers were used for the measurement of fasting blood glucose among the participants.

\section{Definition of terms}

Diabetes mellitus was defined as Fasting Blood Glucose value above $7 \mathrm{mmol} / 1$ (126mg/dl) while Impaired Fasting Glucose (IFG) was fasting blood glucose greater than $6.0 \mathrm{mmol} / 1$ (108mg/dl) but below $7 \mathrm{mmol} / 1$ (126mg/dl). [21] The tuberculosis cases had been previously diagnosed using sputum microscopy and/ or Chest radiography (based on the request of the referring physician) before glucose testing.

\section{Data collection methods}

Fasting Blood Glucose test was repeated on two different occasions for each patient, and overnight fasting was required for all participants, before testing for blood sugar according to international standards. [21] The blood samples obtained from each subject were processed in the laboratory of the State Hospital, Ijebu-Ode.

The plasma glucose levels were assayed in the Chemical Pathology Laboratory using the GLUC-PAP Randox reagent kit manufactured by Randox Laboratory Ltd, United Kingdom. This kit uses the glucose oxidase method. [22] The manufacturer's manual of methodology was strictly followed. Quality control was ensured by using the Randox Multi-sera level 2 and 3 controls for each run.

\section{Ethical Approval}

Ethical permission for the study was obtained from the OOUTH-Health and Research Ethics Committee and the Doctors in Charge of the health facilities where the study was done. Written informed consent was also obtained from all participants before study enrolment.

\section{Data analysis}

Data were analysed using the statistical software (Statistical Package for Social Sciences version 15). Descriptive statistics such as mean, standard deviations, frequencies, and percentages were generated accordingly and presented using tables. Chi-Square test was used to show associations among categorical variables as appropriate. The level of statistical significance was set at $5 \%$.

\section{Results}

A total of 100 subjects were recruited into this study. They were all out-patients attending Primary Health Centres that offered DOTS services. These patients were also classified as Drug-Sensitive TB cases. Out of these 100 subjects previously screened for tuberculosis using sputum analysis for Acid Fast Bacilli (AFB) and as obtained from the case notes, 91.0\% was sputum smear-positive for AFB while $9.0 \%$ was sputum smear-negative for AFB. It was noted from the medical records that those that were sputum smear-negative for AFB had Chest X-ray which radiological features of TB.

Table I shows the socio-demographic characteristics of the subjects with a mean age of $33.4 \pm 9.82$ years and the age groups with the highest frequency included 20-29 years and 3039 years groups. There were more male 
respondents $(61.0 \%)$ than females $(39.0 \%)$. The majority of the study subjects had some form of education as only $2(2.0 \%)$ did not have formal education.

Table I: Socio-demographic characteristics of the subjects

\begin{tabular}{llll}
\hline Parameters & & Frequency & Percentage \\
\hline Age (years) & $\leq 19$ & 3 & 3.0 \\
& $20-29$ & 35 & 35.0 \\
& $30-39$ & 36 & 36.0 \\
& $40-49$ & 18 & 18.0 \\
& $50-59$ & 5 & 5.0 \\
Sex & $\geq 60$ & 3 & 3.0 \\
& Male & 61 & 61.0 \\
Education & Female & 39 & 39.0 \\
& & & \\
& None & 2 & 2.0 \\
& Primary & 34 & 34.0 \\
Marital status & Secondary & 48 & 48.0 \\
& Tertiary & 16 & 16.0 \\
& Single & 36 & 36.0 \\
& Married & 63 & 63.0 \\
Socioeconomic & Divorced & 1 & 1.0 \\
status & Professional & 16 & 16.0 \\
& & & \\
& Skilled & 44 & 44.0 \\
& Unskilled & 25 & 25.0 \\
Religion & Unemployed & 15 & 15.0 \\
& & & \\
& Christianity & 54 & 54.0 \\
& Islam & 45 & 45.0 \\
& Traditional & 1 & 1.0 \\
\hline
\end{tabular}

Table II shows the age distribution of normoglycaemic TB cases in a total of 69 subjects. The age group 20-29 years had the highest frequency of patients with normal blood glucose levels but these findings were not statistically significant.

Table III shows the age distribution of TB patients with DM. There were 11 patients diagnosed by physicians and already commenced on oral hypoglycaemic agents. Therefore, the prevalence of $\mathrm{DM}$ among $\mathrm{TB}$ patients was $11.0 \%$. The age group with the highest proportions in this class of patients was
30-39 years, but this was not statistically significant.

Table IV shows the group of 20 TB patients with Impaired Fasting Glucose Tolerance (IFG) and the age and sex distribution for this category of patients. As such the prevalence of IFG among the TB patients in this study was $20 \%$. Lastly, among these individuals with IFG, there were more males than females, and the age group 2029 years was seen to have a larger number of patients with IFG values without statistically significant difference.

Table V shows that 69 TB cases $(69.0 \%)$ had normoglycaemia (42 males and 27 males) while 
11 TB cases $(11.0 \%)$ had DM (6 males and 5

IFG (13 males and 7 females).

females). In addition, 20 TB cases $(20.0 \%)$ had

Table II: Age and sex distribution of tuberculosis cases with normal blood sugar levels (n=69)

\begin{tabular}{ccccc}
\hline $\begin{array}{c}\text { Age } \\
\text { (years) }\end{array}$ & Sex & $\begin{array}{c}\text { Total } \\
\text { Frequency }\end{array}$ & Percentage \\
\hline $\mathbf{1 9}$ & Male & Female & & \\
$\mathbf{1 9}$ & 2 & 1 & 3 & 4.3 \\
$\mathbf{2 0 - 2 9}$ & 11 & 15 & 26 & 37.7 \\
$\mathbf{3 0 - 3 9}$ & 9 & 16 & 25 & 36.2 \\
$\mathbf{4 0 - 4 9}$ & 3 & 7 & 10 & 14.5 \\
$\mathbf{5 0 - 5 9}$ & 1 & 1 & 2 & 3.0 \\
$\mathbf{2 6 0}$ & 1 & 2 & 3 & 4.3 \\
Total & 27 & 42 & 69 & 100.0 \\
\hline \multicolumn{5}{c}{ Chi-square $\left(\mathbf{X}^{2}\right)=\mathbf{3 . 9 6} ; \mathbf{d f}=\mathbf{5} ; \mathbf{p}=\mathbf{0 . 5 5 4 6}$}
\end{tabular}

Table III: Age and sex distribution of TB cases with DM

\begin{tabular}{|c|c|c|c|c|}
\hline $\begin{array}{l}\text { Age } \\
\text { (years) }\end{array}$ & Sex & & Total Frequency & Percentage \\
\hline & Male & Female & & \\
\hline $20-29$ & 1 & 1 & 2 & 18.2 \\
\hline $30-39$ & 2 & 3 & 5 & 45.4 \\
\hline $40-49$ & 1 & 1 & 2 & 18.2 \\
\hline $50-59$ & 1 & 1 & 2 & 18.2 \\
\hline Total & 5 & 6 & 11 & 100.0 \\
\hline
\end{tabular}

Table IV: Age and sex distribution of TB cases with IFG

\begin{tabular}{|c|c|c|c|c|}
\hline $\begin{array}{l}\text { Age } \\
\text { (years) }\end{array}$ & Sex & & $\begin{array}{l}\text { Total } \\
\text { Frequency }\end{array}$ & Percentage \\
\hline & Male & Female & & \\
\hline $20-29$ & 2 & 6 & 8 & 40.0 \\
\hline 30-39 & 3 & 2 & 5 & 25.0 \\
\hline $40-49$ & 1 & 3 & 4 & 20.0 \\
\hline 50-59 & 1 & 2 & 3 & 15.0 \\
\hline Total & 7 & 13 & 20 & 100.0 \\
\hline
\end{tabular}

Table V: Glycaemic status according to sex among all TB cases

\begin{tabular}{lllll}
\hline Glycaemic status & & Sex & Total Frequency & Percentage \\
\hline & Male & Female & & \\
Normoglycaemia & 27 & 42 & 69 & 69.0 \\
DM & 5 & 6 & 11 & 11.0 \\
IFG & 7 & 13 & 20 & 20.0 \\
Total & 39 & 61 & 100 & 100.0 \\
\hline
\end{tabular}




\section{Discussion}

The rising global trend of co-morbidities ascribed to epidemiologic transitions has led to further discussions and the need for research on screening for both NCDs and infectious diseases. This applies to diseases such as TB and DM. Patients with chronic communicable diseases such as TB are likely to develop comorbidity with non-communicable diseases generally. [23] This is also evident from the findings in the present study of DM and TB comorbidities among the patients who present at DOTS Centres at Ijebu-Ode, Nigeria. Therefore, an overlap of both infectious and noncommunicable diseases, especially among immune-compromised persons, is emphasized.

The prevalence of DM among TB patients in this study was $11.0 \%$. Though this prevalence rate is higher than figures in some previous studies which quoted a range of $4.1 \%$ to $10 \%$, [18,24] it is, however, in keeping with new findings which reported Nigeria having the highest prevalence of $15 \%$ among sub-Saharan African countries. [25]

The prevalence of DM-TB co-morbidity in the present study is also similar to reports in some studies in other settings, such as a retrospective, cross-sectional, descriptive study involving patients diagnosed with pulmonary $\mathrm{TB}$ in the State of Florida, USA, from years 2009 to 2014. The prevalence of DM was $12 \%$ and this is consistent with the current findings in IjebuOde, Nigeria. [26] A prevalence rate of $12 \%$ for DM was also reported among patients with TB in Lagos, [27] $13.9 \%$ in India, ${ }^{[28]}$ while in contrast, a study in Dhaka reported a prevalence rate of $37 \%$. Interestingly, patients with DM had a higher proportion of Multi-Drug Resistant Tuberculosis (MDR TB) cases. [29]
Concerning age distribution, the mean age of the subjects in this study was $33.4 \pm 9.82$ years with two modal age groups of 30-39 years and 20-29 years. The latter age group also constituted the bulk of the subjects classified as DM cases and IFG cases respectively. There were more males in both groups compared to females.

Research has also shown that the global prevalence of DM had risen in the last decades partly due to demographic and lifestyle changes. [30] This is now consistently observed across Africa nations and other countries with emerging economies. This global burden and projections also apply to the pre-diabetic state, with the prevalence of IFG also varying across regions. In the present study, the prevalence of IFG was $20.0 \%$. In other studies, the prevalence of IFG was reported to be $8.9 \%$ in India ${ }^{[28]}$ while another study in Ethiopia reported 29.6\%. [31] Also, much fewer studies have been done to assess both DM and IFG among TB patients; an example was a Chinese study where the prevalence of IFG was 10\%, [32] and a study in Abuja, Nigeria which reported the prevalence of IFG as $7.8 \%$. [33]

Overall, the findings in the present study agreed with other research findings which underscored the significance of screening for DM and glucose intolerance among populations. The lack of uniform testing algorithms in both primary care and tertiary health facilities is also a cause for concern. The outcome of fewer cases of DM and IFG being reported due to limited active screening, which is also inconsistent among health providers. Screening for DM should be a routine investigation before commencing treatment on TB patients. The creation of awareness of potential TB infection among patients with DM should be advocated among healthcare providers. This is to ensure early detection and management of the disease along 
with tuberculosis treatment. This would, in turn, improve the treatment outcomes of $\mathrm{TB}$ and prevent mortality among patients with these comorbidities.

Lastly, further cohort studies are suggested to screen for those co-morbidities using evolving technological tools, to assess the long term effects of these conditions and also appreciate the role of lifestyle modification given epidemiologic transitions.

\section{Conclusion}

The prevalence of DM and IFG among TB patients was high in this study, although, it still relatively aligned with the findings in some other studies conducted elsewhere. This is important when viewed against the backdrop of evolving demographics and epidemiologic transitions on a global scale. Therefore, screening for DM should be a routine investigation before commencing treatment on TB patients just as awareness of potential tuberculous infection among patients with DM should be advocated among healthcare providers, to ensure early detection and management of the disease along with tuberculosis treatment. It is also recommended that the modal age groups identified in this study, which are the 20-29 year and 30-39 year groups, should be targets for more screening to readily identify TB patients with deranged glucose metabolism.

Finally, more studies on TB and DM coexistence on the African continent are required to contribute more knowledge to literature and help us understand other risk factors and outcomes of these diseases as compared to other countries. These would provide evidence of research interventions for policymaking to aid the management and improvement of treatment outcomes.
Acknowledgment: The authors are grateful to the Nursing Sisters Mrs. AA Ogunyemi, Mrs. AA Adeyani, Mrs. SA Orekoya, and other Staff Nurses at the General Hospital, Ijebu-Ode, Ita-Osu Health Centre, Oke- Oyingbo Health Centre and Iwade-Oke Health Centre. Mr. SA Adebusuyi and Mr. RA Oladapo are also appreciated for their laboratory assistance.

Authors' Contributions: SAA initiated, conceived and designed the research and conducted literature search. AOA participated in data collection and literature review. OO contributed to the study design while AOE reviewed the draft of the manuscript. MGJ and S-IFA participated in data analysis and literature review.

Conflict of Interest: None.

Funding: Self-funded.

Publication history: Submitted 21 September 2018; Revised 19 August 2019; Accepted 12 September 2019.

\section{References}

1. Adogu POU, Ubajaka CF., Emelumadu OF., Alutu COC. Epidemiologic Transition of Diseases and Health-Related Events in Developing Countries: A Review, Am J Med Sci 2015; 5(2): 150-157.

2. Enrico G, Monica SS, Delia G, Matthew B, Peter $\mathrm{M}$, Dorothy $\mathrm{YM}$, et al. The global dynamics of diabetes and tuberculosis: the impact of migration and policy implications. Int J Infect Dis 2017; 56: 45-53.

3. Joao DRF, Katherine O, Ute L, Leonor G, Till S, Ping Z, David C, Lydia EM. IDF Diabetes Atlas estimates of 2014 global health expenditures on diabetes. Diabetes Res Clin Pract 2016; 11(7): 48-4.

4. WHO. Global Tuberculosis Control Report 2018: 30- 31.

5. World Health Organization Diabetes Fact Sheets 2018. 
6. Kegne AP, Amoah AG, Mbanya JC. Cardiovascular complications of diabetes mellitus in sub-Saharan Africa. Circulation AHA 2005; 112(23): 3592-3601.

7. International Diabetes Federation (IDF) Diabetes Atlas. 2017.

8. Nasheeta P, Kegne AP, Motala AA, Mbanya JC. Diabetes in the African Region: an update. Diabetes Res Clin Pract 2014; 103(2): 197-205.

9. WHO Global Report on Diabetes. 2016:6.

10. Ogbera AO, Ekpebegh C. Diabetes Mellitus in Nigeria: The past, present and future. World J Diabetes 2014; 5(6): 905-911.

11. Tukur D, Alhaji A, Shehu AU. A review of population based studies on diabetes mellitus in Nigeria, Sub-Saharan Afr J Med 2016; 3(2): 59-64.

12. Geerlings SE, Hoepelman Al. Immune dysfunction in patients with diabetes mellitus (DM). FEMS Immunol Med Microbiol 1999; 26(3-4): 259-265.

13. Jeon CY, Murray MB. Diabetes mellitus increases the risk of active tuberculosis: a systematic review of 13 observational studies. PLoS Med 2008; 5(7): 1091-1101.

14. Victoria H, Reimar WT, Ole H, Nicholai L. Diabetes in Sub Saharan Africa 1999-2011, Epidemiology and public health implications, a systematic review. BMC Public Health 2011; 11: 564.

15. Fiona $Y$, Julia C, Lucy J, Nigel U. A review of co-morbidity between infectious and chronic diseases in Sub Saharan Africa: TB and Diabetes mellitus, HIV and Metabolic Syndrome, and the impact of globalization. BMC Globalisation Health 2009; 5(9).

16. Restrepo BI, Camerlin AJ, Rahbar $\mathrm{MH}$, Wang W, Restrepo MA, Susan FH, et al. Cross- sectional assessment reveals high diabetes prevalence among newly diagnosed tuberculosis cases. Bull World Health Org 2011; 89(5): 352-359.

17. Satyavani K, Vijay V. Diabetes and Tuberculosis- a co-epidemic of public health importance in the developing world. J Current Sci 2017; 113(7).

18. Mahteme HW, Gunner AB, Solomon AY. Prevalence and associated factors of tuberculosis and diabetes mellitus comorbidity: A systematic review. PLoS One 2017; 12 (4).

19. Kelly D, Richard EC. Tuberculosis and Diabetes mellitus: convergence of two epidemics. Lancet Infect Dis 2009; 9(12): 737746.

20. National Population Commission. Population distribution tables by Sex, State, LGA and Senatorial district, (Updated 2010). Population and Housing Census 2006, Nigeria.

21. WHO. WHO definition and diagnosis of diabetes mellitus and intermediate glycaemia: report of a WHO/IDF consultation. Geneva: 2006.

22. Trinder P. Determination of glucose in blood using glucose oxidase with an alternative oxygen acceptor. Ann. Clin. Biochem 1966; 6 ;24.

23. Olayinka AO, Anthonia O, Yetunde K. Prevalence of diabetes mellitus in persons with tuberculosis in a tertiary health center in Lagos, Nigeria. Indian Journal of Endocr Metab. 2013; 17(3):486-489.

24. Animut A, Amsalu W. Prevalence of diabetes mellitus among tuberculosis patients in Sub-Saharan Africa: a systematic review and meta-analysis of observational studies. BMC Infect Dis 2019, 19:254. 
25. Mkhontfo, Mandzisi Mbongeni. An Examination of the Distribution of Diabetes Mellitus among TB Patients with Pulmonary Tuberculosis and Drug-Resistant Tuberculosis in the State Of Florida, USA. (2016). Graduate Theses and Dissertations. Available at http:/ / scholarcommons.usf.edu/etd/6325. Accessed on October 5, 2018.

26. Ogbera AO, Kapur A, Odeyemi $\mathrm{K}$ et al. Screening for diabetes Mellitus and human immunodeficiency virus infection in persons with tuberculosis. J Prev Med Hyg 2014; 55(2): 42-45.

27. Magna MA, Subrata KP, Subhashisa S, Sandipana P, Sanghamitra P. Diabetes Mellitus among Newly Diagnosed Tuberculosis Patients in Tribal Odisha: An Exploratory Study. J Clin Diagn Res 2016; 10(10): 06-08.

28. Tanzila T. A survey on the prevalence of diabetes in tuberculosis patients in NIDCH hospital, Dhaka. EWU Repos, 2016. Available at http:/ / dspace.ewubd.edu/handle/2525/170 7. Accessed on 22nd September 2018.
29. Assefa G, Solomon M, Shiatye A, Hanan Y. High magnitude of diabetes mellitus among Active Pulmonary Tuberculosis Patients in Ethiopia. BR J Med Med Res, 2014, 4(3): 862872.

30. Shugang L, Shuxia G, Fei H, Mei Z, Jia H, Yizhong $\mathrm{Y}$, et al. Prevalence of Diabetes Mellitus and Impaired Fasting Glucose, Associated with Risk Factors in Rural Kazakh Adults in Xinjiang, China. Int J Environ Res Pub Health 2015; 12(1): 554-565.

31. Lawson L, Muc M, Oladimeji O, Iweha C, Opoola B, Abdurhaman ST, et al. Tuberculosis and diabetes in Nigerian patients with and without HIV. Int J Infect Dis 2017; 61: 121-125.

32. UNGA Third UN level high meeting on Non-Communicable Diseases 2018.

33. Ben JM, Knut L, Stephen DL, Giovanni BM, Peter M, Philippe G, et al. Tuberculosis comorbidity with communicable and noncommunicable diseases: integrating health services and control efforts. Lancet Infect Dis 2013; 13(5):

436-448.

\begin{tabular}{|c|c|c|} 
This is an Open Access document licensed for distribution under the terms and conditions of the Creative \\
Commons Attribution License (http://creativecommons.org/licenses/by-nc/4.0). This permits unrestricted, \\
non-commercial use, reproduction and distribution in any medium provided the original source is adequately \\
cited and credited.
\end{tabular}

